\title{
An evolutionary perspective on paranoia
}

4

Nichola J Raihani ${ }^{1} \&$ Vaughan Bell ${ }^{2}$

6

1. Department of Experimental Psychology, University College London, 26 Bedford Way, WC1H $0 \mathrm{AP}$

\section{Abstract}

Although paranoia is the most commonly presenting symptom of psychosis, paranoid thoughts occur frequently in the general population and range widely in severity, from mild socio-evaluative concerns to frank delusions about the harmful intentions of others. Furthermore, paranoia commonly appears after a surprisingly diverse range of difficulties including trauma, brain injury, sleep deprivation, drug use, and psychiatric and neurological disorder. Evolutionary accounts of paranoia have been proposed before but have largely focused on paranoia as a misplaced threat response. Although social threat is clearly a key component, the experience of paranoia is markedly more complex than these accounts would lead us to believe: paranoia can involve multiple alterations in the perception of the social environment, the identification of specific but seemingly arbitrary groups as the source of persecution, and extended beliefs about conspiracy and complex coordination between the perceived persecutors. Here, we argue for an evolutionary approach to paranoia that more fully accounts for its complex social phenomenology and considers how it can be understood in light of our evolved social cognition. More specifically, in terms of the ability to form coalitions and coordinate between groups in situations of cooperation and competition. 
29 Evolutionary accounts of paranoia have been proposed before $(1,2)$ but have largely focused on 30 paranoia as over-sensitive or misplaced social threat perception (1). Given that misperceiving social threat would seem to be a serious problem for forming and maintaining productive social relationships, this article focuses on an evolutionary account of why paranoid thinking is such a common human characteristic and why paranoia can become intense and disabling after many forms of social, psychological and neurological difficulties.

\section{Current conceptualisation of paranoia}

A persecutory belief is considered to be the central defining feature of paranoia and includes two essential elements: i) a belief that harm will occur, and ii) an attribution that others intend this harm (3). In the general population, such persecutory ideas can be experienced with varying degrees of frequency and entertained to varying degrees of intensity. Paranoia can range from mild thoughts about others' intentions to beliefs that are sufficiently unlikely, and inflexible to be classified as a psychiatric symptom, most notably, as a paranoid delusion (4). One of the implicit assumptions about paranoia is that it represents an exaggerated or false attribution of harmful intent to others. However, given the continuum of paranoia, paranoid explanations can, and occasionally should, be accurate (e.g. see $(5,6))$ although these are likely to be increasingly inaccurate as paranoia becomes more becomes more disabling and a likely focus of clinical concern $(7,8)$.

Epidemiological studies show that paranoia shows full taxometric continuity throughout the population, indicating that categorical distinctions used in psychiatric diagnosis are not reflected in a clear point of change of severity in the population (9-11). Nevertheless, this continuous distribution in the population does not necessarily imply that underlying causes are fully continuous within individuals, over time, or between sub-groups (12). Most current research has focused on paranoia in the context of mental health, typically in people without individually diagnosable neurological disorder, and has identified various risk factors and cognitive process that support paranoid thinking. Indeed, paranoia has now been reliably associated with living in areas of low social cohesion (13), worry (14), sleep deprivation $(15,16)$, early life adversity and abuse, trauma (17) and victimisation $(16,18)$. Paranoia has also been found to co-occur with general cognitive biases relating to causal and probabilistic reasoning and belief flexibility $(19,20)$. However, diagnosable paranoid states can also be caused by a wide range of direct disturbances to brain function. Paranoia is common in psychosis following epilepsy (21), brain injury $(22,23)$ and dementia (24). It is also one of the most frequent unwanted side-effects for several classes of recreational drugs (25-27). Indeed, in terms of the causes and contexts in which it appears, paranoia is perhaps most remarkable for being associated with such a wide range of difficulties, impairments and stresses. 
Given this diversity, the aim of this article is to ask whether paranoia might sometimes serve an adaptive (fitness-enhancing) function and how an evolutionary perspective can help us to predict where paranoia will be most common. To be clear, our aim is not an attempt to explain how frank paranoid delusions and - by extension - psychotic spectrum disorders, have been favoured by selection. Indeed, based on the lowered reproductive success of individuals with these disorders and the lack of evidence of benefits to kin (see (28)), we think that this is highly unlikely. Our overarching hypothesis is that the existence of paranoia can generally be understood as a consequence of selection for detecting and evaluating coalitional threat. We first describe the phenomenology of paranoia and argue that current evolutionary theories do not fully account for the perception of conspiracy and selective identification of arbitrary persecutors that are so common in paranoia. We suggest that coalitionary competition, which can occur both within and between groups and which can be relatively stable in some contexts and yet highly flexible in others, can help to explain why paranoia takes the form it does. Our hypothesis predicts that within-individual variation in paranoid thinking should occur in response to immediate context-specific changes in the perception of coalitionary threat (as defined by (29)), whereas stable between-individual differences in paranoia are likely to emerge in response to chronic threat from others. Finally, we explore why impairments to brain function also commonly predispose individuals to paranoia, and whether this is likely to be an adaptive response to the environment or a maladaptive consequence of cognitive constraints.

\section{Understanding the full social phenomenology of paranoia}

Freeman and Garety's (3) definition has been useful in providing a clear operational definition of a central component of paranoia. However, existing approaches to paranoia have tended to conceptualise paranoia in terms of cognitive processes used to make sense of other individuals rather than groups. One limitation of this approach is that it fails to account for why the experience of more severe paranoia often involves the misperception of group boundaries and collective action. Indeed, paranoia is frequently accompanied by other features that are common enough to be included in phenomenological descriptions, both historical and modern, but are often neglected by more recent cognitive approaches. These are i) the perception of a conspiracy behind the intentional harm, and ii) social selectivity in terms of identifying the people perceived to be the source of intentional harm.

Conspiracy thinking is common in the general population (30) and is defined as a tendency to provide "explanations for important events that involve secret plots by powerful and malevolent groups" (31)). In paranoid delusions, however, conspiracy thinking often becomes self-focused, with delusions commonly involving the perception of organised attempts to harm the believer, rather than malign explanations for public events. The perception of a self-focused conspiracy has been identified as a central characteristic of delusional paranoia from early in the history of psychiatry (32) and forms part of many modern phenomenological descriptions $(33,34)$. Cameron (1959) conceptualised this aspect 
102 of paranoia as a belief in a persecutory 'pseudo-community' who are perceived to be united in a co-

103 ordinated undertaking against the paranoid individual but who fail to correspond to any group in

104 wider society who share the coordinated aims and actions attributed to them. Unlike public conspiracy

105 theories, these concerns are more likely to focus on the history, intentions and day-to-day activities of

106 the believer.

107

108 Although paranoia involves a belief that others intend harm to the believer, these concerns typically

109 pertain to specific individuals or social groups and also commonly involve the misperception of group

110 boundaries and coordinated group action. In increasingly severe paranoia, these concerns and

111 misperceptions become increasingly exaggerated and may present as frank persecutory delusions.

112 Studies of delusional patients indicate that the majority selectively identify specific groups as

113 responsible for their maltreatment. In a study of delusions in Korean, Korean-Chinese, and Chinese

114 patients conducted by Kim et al. (35), only $27.4 \%, 17.7 \%$ and $24.6 \%$ of persecutors, respectively,

115 were unspecified, while the rest were variously identified as groups such as relatives, neighbours, the

116 police, or medical personnel (see also (36)). Green et al. (37) reported that persecutory delusions

117 could be classified as focusing on individuals (e.g. "my father"), groups with defined members ("[the

118 patient's] neighbour, his neighbour's brother and mates"), established social groups ("the police"),

119 undefined groups ("people", "spirits") and all others ("everyone") with perceived individual and

120 multiple persecutors each consisting $50 \%$ of the total.

121

122

Evolutionary approaches to paranoia

123 Attempts to answer the question of why some people are more paranoid than others have typically

124 appealed to proximate level explanations (Box 1) such as genetics, life history or cognitive biases (4).

125 Nevertheless, these approaches do not answer the issue of why we have a cognitive capacity for

126 paranoid thinking (Box 2) and whether between-individual variation in paranoid thinking might, in

127 some environments, be selectively advantageous in fitness terms. From a Darwinian perspective, a

128 fearful response to danger, whether actual or potential, is likely to carry significant fitness benefits

129 and to have been subject to strong selection in many species (38). Nevertheless, not all individuals

130 show an equivalent magnitude of response to the same threatening stimulus or context: levels of

131 fearfulness differ markedly across individuals, even within a species. The question of how stable,

132 between-individual differences in fearful responses might arise and be stabilised by selection falls

133 under a broader banner of research on the evolution of stable behavioural types. Research in this field

134 has shown that the evolution of variation in behavioural types stems from trade-offs in pursuing

135 different fitness-relevant activities. For example, investing in growth (e.g. via foraging) often comes

136 with an attendant increased risk of predation $(39,40)$ and so strategies aimed at increasing growth are

137 likely to be traded-off against strategies that reduce predation risk. Organisms must therefore balance

138 the rewards of investment in growth against the increased mortality risk; the optimal resolution of 
such trade-offs in different environments or for different individuals can therefore select for variation in fearfulness, aggression, risk appetite and so on, which broadly dictate individual life history strategies and associated behaviour.

In addition to balancing such trade-offs, organisms must also effectively manage costs from errors that occur due to perceptual uncertainty ('error management theory', (41), Box 3). Specifically, error management theory predicts that when there are asymmetries in the costs of false-positive and falsenegative error types, then selection will favour strategies that minimise the chance of making the costlier error, even if this produces many behavioural mistakes. Following the logic of error management theory, previous evolutionary accounts $(1,41)$ have suggested that paranoia is an evolved psychological mechanism shaped by the selective pressures of catastrophic harm from others that is tuned to have a low threshold for detecting social threat. Individual variation in the relative asymmetry of error types is proposed to account for variation in paranoia across the full spectrum (see Box 3 for a critique).

\section{Shortcomings of existing evolutionary theories: accounting for paranoia beyond social anxiety}

Nevertheless, existing evolutionary theories of paranoia based solely on social threat detection do not fully account for the complex phenomenology of paranoia. Specifically, we have to ask why a mechanism aimed at detecting and avoiding social threats does not solely result in variation in avoidance and/or submissive and appeasement behaviours (as is also observed in many non-human species, see (42) and also discussed elsewhere, $(43,44))$, but also incorporates more complex features that are not adequately explained by this approach. Namely, selective identification of a specific (yet often seemingly arbitrary) group of persecutors, the attribution of unobservable malign intentions and motives to these individuals, and the formulation of hypothetical narratives rendering these attributions subjectively plausible. Below, we focus on the first of these features but see Box 2 for a discussion of the evolution of inferential causal reasoning abilities (including mental state attribution) in humans.

An important feature of human social groups is the presence of coalitions: any situation where two or more individuals unite in competition against a third party or parties $(45,46)$. Coalitionary conflict in human groups can manifest in the form of lethal aggression ('lethal raids' reviewed in (47)) but can also include non-lethal and non-aggressive conflict, such as stigmatization, ostracism, exclusion, and derogation. For example, witchcraft accusations have been (and still are) used to identify individuals or groups for ostracism, persecution or even death $(48,49)$. In modern industrialised societies, similar forms of indirect aggression are used by coalitions to damage the reputation of (often higher-ranking) rival, for example via gossip or derogation (see $(50,51))$. 
176 This persistent risk of persecution selects for what Boyer (29) has named a 'coalitional psychology'

177 that anticipates and deflects these threats by integrating oneself within a coalition or coalition(s),

178 recognising and categorizing others as allies or potential competitors; and using these categorizations

179 to predict how others might behave or react in specific social interactions (29,52-55). One might

180 expect social threat detection mechanisms to be sensitive to reliable indicators of coalitional threat,

181 such as dominance hierarchies, signals of group membership and the cohesiveness of rival coalitions

$182(29,53)$ and, accordingly, experimental evidence shows that exposing people to these different forms of social threat does increase the tendency to make paranoid attributions $(56,57)$.

185 Nevertheless, paranoia often involves the selective identification of a (seemingly arbitrary) group of 186 persecutors, where malign intent is attributed to some individuals (or groups) but not others (e.g. 'I'm 187 being persecuted by the CIA' [and not FBI] or 'I'm being persecuted by my family' [but not my neighbours]'). We suggest that this arbitrary selectivity might reflect the fact that coalition boundaries in human groups are themselves highly fluid and flexible and can be formed in the absence of any stable group identifiers (58). The fact that coalitions can be formed on the basis of minimal cues or markers of similarity in turn selects for cognitive machinery that readily and flexibly categorizes people into groups on the basis of such 'minimal' cues $(59,60)$. Indeed, humans readily form and detect minimal groups, even from a young age (60) and the perception of these groups fundamentally alters expectations about the intentions and behaviour of individuals within them (reviewed in (61)). Assuming that paranoia builds on this existing cognitive machinery helps to explain the seemingly arbitrary selectivity in the identification of perceived persecutors. This raises an interesting theoretical question as to the extent to which increasingly severe paranoia reflects variation in cognitive processes involved in perceiving coalitions and alliances, as opposed to processes involved in the attribution of (harmful) intent to others. We suggest that disambiguating these processes and how they vary across the paranoia spectrum will be a fruitful avenue for further research.

\section{Predictions deriving from a coalitional psychology model of paranoia}

203 A coalitional perspective suggests that variation in paranoia could function to protect individuals from coalitionary threat in specific contexts and therefore serve an adaptive function when either the probability and/or the costs of harm from others are high. A prediction of this hypothesis is therefore that variation in paranoid thinking will reflect the background probability and/or costs of coalitional conflict. Epidemiological evidence supports this prediction: an increased tendency for paranoid thinking has been documented in general population groups that are involved in higher-than-average rates of coalitionary aggression, such as gang members (62) and army veterans $(63,64)$. The probability of inter-coalitionary violence is increased under conditions of resource scarcity (65) and, as expected, living in poverty is also associated with increased tendency for paranoid thinking (66). 
213 Variation in paranoia should also be sensitive to the perceived costs of receiving inter-coalitionary

214 aggression, which escalate with low coalitionary support, low social rank or increasing power

215 imbalances between coalitions $(67,68)$. In support of this prediction, risk for psychosis (for which

216 paranoia is the most common delusional theme) is higher among people who have small social

217 networks (69) or who are socially isolated, both of which are proxies for low coalitionary support.

218 Epidemiological evidence supports the idea that perceived power imbalances can raise the risk for

219 psychosis and, by extension, can also increase the probability for paranoid thinking. For example, low

220 social rank (both perceived and objective) is an important predictor for increased paranoia (70) - a

221 finding that has recently been supported by experimental work where participants' social status

222 relative to that of a partner was experimentally manipulated (56). Similarly, being part of a

223 marginalised social group (e.g. a low status immigrant, or an ethnic minority) is a risk factor for

224 paranoia (71), which can be ameliorated by living in increased densities within the marginalised

225 group (72). A coalitional psychology perspective on paranoia would predict this otherwise

226 paradoxical 'ethnic density effect' since living at higher ethnic densities with perceived coalition

227 members should be associated with an increased perception of coalitionary support.

228

229 Paranoia also varies within individuals and is fine-tuned to the degree of coalitional threat in the

230 current interaction. For example, experimental work where people interact with a political affiliate or

231 with a political adversary shows that harmful intent attributions, the fundamental component of live

232 paranoid ideation (Box 4) are stronger for the dissimilar than for the similar interaction partner, as

233 expected (56). Paranoid thinking should also respond flexibly to the cohesiveness of coalitions since

234 cohesive coalitions are more able to work together to harm rivals (29). As expected, recent work has

235 shown that paranoid attributions increase when participants interact with a cohesive pair of opponents

236 compared to a pair of non-cohesive opponents (57). Thus, observational and experimental evidence

237 suggests that paranoid thinking is flexible and responsive to social context in both the short and long-

238 term, as would be expected if paranoia is the output of a mechanism for detecting and avoiding

239 coalitionary threat.

240

241 Paranoia also varies widely across the lifespan, emerging in adolescence, being most pronounced in

242 early adulthood (73) and declining as individuals age (74). Indeed, if paranoia is an output of a

243 coalitional psychology, then its emergence should coincide with onset of coalitional threat. Empirical

244 evidence suggests that coalitional competition begins to emerge when individuals reach puberty and is

245 most intense during late adolescence and early adulthood (75). Competition during adolescence may

246 play an important role in the formation of and integration into coalitions that ultimately determine

247 individuals' status, access to resources (including mates) and reproductive success. In modern tribal

248 societies, such as the Nyangatom, men form close alliances with same-age individuals during

249 adolescence. It is also at this time that men begin to join lethal raiding excursions to neighbouring 
groups (usually with members of their coalition), continuing to participate in these raids until they end their reproductive careers (c. age 45, (76)). More generally, interaction with peers increases markedly during adolescence (77), leading also to an increase in social competition at this age. For example, bullying - which can be construed as a form of coalitional competition - is prevalent across all world cultures (and also in pre-industrialised societies) and increases in frequency as children enter adolescence (78), peaking around the age of 14 (79). Other work has shown that adolescence is a period that is characterised by increased sensitivity to social threat, social risks and social exclusion (80-82), as well as being a common onset period for many mental health problems, including psychotic-spectrum disorders $(73,83)$. Thus, we suggest that the developmental trajectory of paranoia reflects a selective process that balances sensitivity to threat in line with fitness-relevant outcomes. Individuals may also experience sensitive periods during development, where cues from the (social) environment exert exaggerated effects on subsequent development. Sensitive periods are expected to evolve whenever the early environment can reliably predict future conditions and when there are constraints on plasticity (84). The conditions experienced during a sensitive period of development can act as a 'weather forecast', guiding subsequent development along different trajectories and generating adaptive matches between the environment and the individual's phenotype (84-88). It has been suggested that adolescence could be one such sensitive period in development $(84,89,90)$, with the evolutionary relevance being that individuals receive more reliable cues about the kind of social world they will inhabit and their place in it during adolescence than earlier in development (see (84)). One of the key outstanding questions with respect to paranoia will be to determine whether social threat shapes responses across the lifetime, or whether there are sensitive periods of development during which exposure to social threat exerts lasting consequences on social cognition and behaviour. If the latter, then identifying when these sensitive periods are and how they vary in response to the stochasticity of the social environment (e.g. $(88,91))$ will also be fruitful.

When does paranoia become pathological?

277 Having argued so far in favour of viewing variation in paranoia as part of a normally-functioning, 278 naturally selected human psychology, we now address the question of when paranoia might be best viewed as a disorder and, therefore, under negative selection. The definition of mental disorder is historically controversial and beyond the scope of this article: here we adopt the 'Harmful Dysfunction' definition proposed by Wakefield (92) which states that a) mental disorders are conditions that cause harm to the person as judged by the standards of the person's culture, and b) that the condition results from the inability of some internal mechanisms (psychological or physiological) to perform its natural function, wherein a natural function is an effect that is part of the evolutionary explanation of the existence and structure of the mechanism. Importantly, as with many other 
that demarcate the boundary between ordered and disordered paranoia (93) without denying clear pathology within this range.

An analogy may be helpful: fever helps the body fight off pathogens and can therefore be viewed as part of a normally-functioning body's evolved responses to infection. Nevertheless, the underlying mechanisms regulating temperature can become impaired or fail, leading to increasingly dysregulated fever that can sometimes be fatal. Clearly, in the latter case, fever would be viewed as pathological (i.e. disordered) despite that fact that, under normal circumstances, fever is an adaptive response to infection. Based on this logic, we suggest that as paranoia becomes increasingly severe and therefore less responsive to threat in the immediate environment, it is increasingly likely to stem from dysfunction in the underlying cognitive mechanisms that support threat evaluation and so is likely to fit the definition of disorder (being, by implication, maladaptive). We remain agnostic about the precise cut-off point for separating ordered from disordered paranoia, as well as about the magnitude and linearity / non-linearity of fitness costs involved.

At this point however, it is also instructive to raise another question. Paranoia is increased by a wide range of brain injuries and impairments, including substance use and abuse, sleep deprivation, traumatic head injury, and dementia: do these impairments imply that the resulting paranoia is necessarily disordered? We argue that it need not be the case. Rather, we suggest that it is possible that increased paranoia in response to brain impairment reflects the correct functioning of a "cognitive failsafe' because cognitive impairment renders people at higher risk of being exploited by others whom were previously allies or makes them less able to incur the costs of being exploited (e.g. see $(94,95))$ and therefore a bias toward developing paranoia, rather than other socio-affective states, after impairment may have a protective effect. Thus, this might constitute an adaptive response rather than a disorder. Nevertheless, following the fever analogy above, this hypothesis allows that in some individual contexts, impairments to the mechanisms of the cognitive failsafe can lead to increasingly severe and disordered paranoia, resulting in worse or even catastrophic outcomes for an individual.

\section{Conclusions}

316 We argue that an evolutionary approach can help make sense of otherwise puzzling features of paranoia. These include a population continuum of paranoia that includes both context-sensitive paranoid thinking and inflexible, unlikely paranoid delusions, as well as the tendency to selectively identify seemingly arbitrary groups of persecutors, and to perceive that one is the target of conspiracy. We also note that our approach highlights some key areas of future research. The first is on the phenomenology of paranoia and we suggest that the content of delusions in severe paranoia should

322 often reflect common sources of coalitionary threat (e.g. coordinated groups and cliques, higher status 323 individuals, physical harm, threats to reputation). For some individuals, different threats may be more 
salient or more likely and this might well be reflected in the content of delusions across individuals (e.g. see (96)). Secondly, we suggest additional focus is needed on how people perceive social groups, including processes relating to identification with in-group and categorising others as out-group, and how these processes may be altered in people experiencing severe paranoia. We also note that paranoia has received surprisingly little attention from evolutionary scientists in comparison to other psychiatric difficulties and we hope it becomes of further interest in the field, given its clear relevant to fitness concerns, its diverse presentation and ubiquity in human history.

\section{Box 1. Proximate and Ultimate level explanations}

It is worth clearly delineating between proximate and ultimate levels of explanation. In evolutionary biology, an answer to the question of 'why' an individual behaves in a certain way can take two broad, non-mutually exclusive forms: proximate and ultimate level explanations (97-100). Ultimate level explanations provide the answer to 'why' the behaviour exists: they describe the function of the behaviour in question and show how such behaviour, on average, is associated with fitness increases. Proximate level explanations, on the other hand, are concerned with 'how' the behaviour is implemented. For example, proximate level explanations could describe the psychological mechanisms that support or constrain the behaviour but could also include the hormonal or physiological basis of behaviour. For example, one might answer the question of why a lioness chases a zebra by saying that the lioness needs to eat and is motivated by hunger, or that she has babies to feed, or that she is joining the other lionesses in the pride in the hunt - these would all be valid proximate-level explanations. An ultimate level explanation for hunting behaviour is that lionesses who attempt to hunt and kill prey have more surviving offspring than those who do not partake in hunting and so this behaviour has been selected for in lion populations over evolutionary time. Clearly, the two explanations are not mutually exclusive. However, a proximate level answer cannot be posed as the solution to an ultimate question of why behaviour exists.

350

\section{Box 2. Which features of paranoia are unique to humans and why?}

352 Evidence for the sort of inter-coalition competition that we propose results in selective pressure for variation in paranoia is also present for other species, raising the question of to what extent features of paranoia may be present in non-humans animals. For example, lethal intergroup competition in the form of lethal raiding occurs also in chimpanzees(47), and more subtle forms of coalitional competition have also been observed in many other social non-human species (see (46) for a review). There is also convincing evidence for variation in social anxiety in non-human species (42). However, we would argue that the key cognitive mechanism that underlies the ability for paranoid thinking: namely the ability to reason about unobservable causal mechanisms to explain why events have occurred in the past or might occur in the future seems to be, for the most part, unique to humans (101). The ability to attribute intentions to others (also key in paranoia and arguably absent in non- 
human species, (102)) might represent an instantiation of this ability for inferential causal reasoning, albeit one that is specific to the social domain (103). The question of what selective pressures are most likely to have favoured the human-specific propensity to seek diagnostic causal explanations for phenomena humans is hotly debated (see $(103,104))$ and a full discussion is beyond the scope of this article. Specifically, it remains an open question whether the human tendency to seek and draw causal inferences evolved in response to social selection pressures, or whether this is more likely to have evolved in response to ecological selection pressures, being subsequently co-opted and used in the social domain.

\section{Box 3. Error-management theory}

Error management theory (41) states that the existence of asymmetric error costs can favour the evolution of strategies that err on the side of caution, thereby protecting individuals from catastrophic errors, and may be presented as cognitive biases - that is, psychological mechanisms that result in inaccurate perceptions of the true environment but that can shape behaviour in on-average beneficial ways (see (105-108) for discussion). For example, it may be better to mistake a stick for a snake, than a snake for a stick, because the latter mistake is more likely to be fatal. False alarms of this sort are abundant in nature, in humans and non-human species $(28,41)$. Crucially, selection is not expected to produce perfectly optimal behaviour under all circumstances but rather to produce strategies that are on average successful over the lifetime and within a population. From an evolutionary perspective, many behavioural 'mistakes' (mistaking sticks for snakes) would be permitted under a broadly adaptive strategy of 'all snake-shaped things should be initially treated as if they could be snakes'. The strength of such biases (whether behavioural or cognitive) should therefore reflect the asymmetry in error costs: the greater the risk that one error type will produce a catastrophic outcome in comparison to the other, the more likely individuals are to be biased towards making the least costly of the error types. Nevertheless, it is worth noting a shortcoming in the typical application of error management theory to paranoia: in social groups, the asymmetric costs in terms of misperceiving social motivations may depend on context (41). The costs of wrongly treating someone as trustworthy who actually wants to do you harm may be severe. However, the costs of wrongly treating a coalition member as untrustworthy may also be severe due to the fact non-cooperation often results in reciprocal defection (109), punishment $(110,111)$, or exclusion $(112,113)$. Indeed, mistakenly treating others as if they might harm you can jeopardize the future of potentially mutually-beneficial partnerships, to the extent that the costs associated with such errors have been posited as the basis for the extraordinarily high levels of human trust and cooperation in seemingly anonymous, one-shot interactions (when the potential for cheating and being exploited is rife) (114) (but see (115)). So, while it may be adaptive to consistently err on the side of misperceiving a snake for a stick - as in the traditional formulation of error management theory - the costs are highly asymmetric in comparison to human threat examples in large part because you cannot form a coalition with a snake or 
incorrectly reject it as an ally. Importantly, the exact distribution of cost asymmetry that drives selection in these situations is an empirical question and it is possible that the costs of underperceiving hostile intent in others is still on overage higher than the costs of over-perceiving hostile intent in allies. However, the fact that the latter is well-established as having costs in human social groups suggests that cost asymmetry will not mirror contexts that are most commonly cited as selective pressures that drive the evolution of cognitive biases (sticks, snakes etc).

\section{Box 4. Measuring paranoia in experiments involving genuine social interactions}

Paranoia by definition affects how we form and update impressions of others in social interactions. It is therefore instructive to attempt to measure paranoia in settings where participants experience genuine social interactions with others. Game theory tasks - typically used in experimental and behavioural economics - provide many paradigmatic examples of stylized social interactions that can be used to infer or measure social behaviour and preferences and these tasks are now being used to great effect to better understand how social cognition and behaviour vary in paranoia. Many game theoretic tasks operationalise pro-social behaviour as the willingness to forego financial earnings in the task in order to benefit the partner(s) in the interaction. Games can be one-shot or repeated, occur among pairs or groups of individuals and allow for various forms of social behaviour, including cooperation and punishment. In particular, many game theoretic tasks allow us to measure paranoid attributions since the motives underpinning the decisions to cooperate or not in these tasks are often murky. Consider, for example, the Dictator Game. In this two-player game, one person (the 'dictator') is given a sum of money and can choose whether to send some to the partner (the 'receiver') or to keep all the money for themselves. The receiver has no active role in this game and must accept whatever share the dictator offers. Importantly, the motives underpinning a dictator's decision to keep all the money are ambiguous. One might infer that the dictator is motivated by greed (or self-interest). Alternatively, one might also infer that the dictator is motivated by a desire to deny the receiver any money (i.e. intent to harm). Inferring harmful intent in such an interaction is a reliable proxy for paranoid thinking and, in a series of studies using participants from the general population $(56,57,116)$, it has been shown that people who have higher tendency for paranoid thinking make stronger harmful intent attributions in these tasks. The degree to which individuals attribute harmful intent to others in turn predicts their willingness to punish their interaction partners (117). 
430 Acknowledgements

431 NR is funded by a Royal Society University Research Fellowship; VB is supported by a Wellcome

432 Trust Seed Award in Science [200589/Z/16/Z]. Thanks to Louise Barrett for helpful comments on an 433 earlier draft.

434

435 
Green MJ, Phillips ML. Social threat perception and the evolution of paranoia. Neuroscience \& Biobehavioral Reviews. 2004;28(3):333-42.

Veras AB, Souza TGE, Ricci TG, de Souza CP, Moryiama MC, Nardi AE, et al. Paranoid Delusional Disorder Follows Social Anxiety Disorder in a Long-Term Case Series: Evolutionary Perspective. The Journal of Nervous and Mental Disease. 2015 203(6):477-9.

Freeman D, Garety PA. Comments on the content of persecutory delusions: Does the definition need clarification? British Journal of Clinical Psychology. 2000;39(4):40714.

Freeman PD. Persecutory delusions: a cognitive perspective on understanding and treatment. The Lancet Psychiatry. 2016 ;3(7):685-92.

Jack A, Egan V. Paranoid thinking, cognitive bias and dangerous neighbourhoods: Implications for perception of threat and expectations of victimisation. International Journal of Social Psychiatry. 2015;62(2):123-32.

6.

Jun H-J, Nam B, Fedina L, Smith ME, Schiffman J, Link B, et al. Paranoid beliefs and realistic expectations of victimization: Data from the survey of police-public encounters. Schizophrenia Research. 2018 (in press) structure of paranoia in the general population. The British Journal of Psychiatry. 202(6):419-27.

9.

Bell V, O'Driscoll C. The network structure of paranoia in the general population. Social Psychiatry and Psychiatric Epidemiology. 2018;53(7):737-44.

Elahi A, Algorta GP, Varese F, McIntyre JC, Bentall RP. Do paranoid delusions exist on a continuum with subclinical paranoia? A multi-method taxometric study. Schizophrenia Research. 2017; 190: 77-81.

van Os J, Linscott RJ, Myin-Germeys I, Delespaul P, Krabbendam L. A systematic review and meta-analysis of the psychosis continuum: evidence for a psychosis proneness-persistence-impairment model of psychotic disorder. Psychological Medicine. 2009;39(2):179-95.

Taylor MJ, Freeman D, Ronald A. Dimensional psychotic experiences in adolescence: Evidence from a taxometric study of a community-based sample. Psychiatry Research. $2016 ; 241: 35-42$.

David AS. Why we need more debate on whether psychotic symptoms lie on a continuum with normality. Psychological Medicine. 2010;40(12):1935-42.

Freeman D, McManus S, Brugha T, Meltzer H, Jenkins R, Bebbington P. Concomitants of paranoia in the general population. Psychological Medicine. 2011;41(05):923-36.

Startup H, Freeman D, Garety PA. Persecutory delusions and catastrophic worry in psychosis: Developing the understanding of delusion distress and persistence. Behaviour Research and Therapy. 2007;45(3):523-37. 
15. Kahn-Greene ET, Killgore DB, Kamimori GH, Balkin TJ, Killgore WDS. The effects of sleep deprivation on symptoms of psychopathology in healthy adults. Sleep Medicine. 2007;8(3):215-21.

16. Bird JC, Waite F, Rowsell E, Fergusson EC, Freeman D. Cognitive, affective, and social factors maintaining paranoia in adolescents with mental health problems: A longitudinal study. Psychiatry Research. 2017;257:34-9.

17. Bentall RP, Wickham S, Shevlin M, Varese F. Do Specific Early-Life Adversities Lead to Specific Symptoms of Psychosis? A Study from the 2007 The Adult Psychiatric Morbidity Survey. Schizophrenia Bulletin. 2012;38(4):734-40.

18. Catone G, Marwaha S, Kuipers E, Lennox B. Bullying victimisation and risk of psychotic phenomena: analyses of British national survey data. The Lancet Psychiatry. 2015;2(7):618-24.

19. McLean BF, Mattiske JK, Balzan RP. Association of the Jumping to Conclusions and Evidence Integration Biases With Delusions in Psychosis: A Detailed Meta-analysis. Schizophrenia Bulletin. 2016; 43: 344-354.

20. Buchy L, Woodward T, Liotti M. A cognitive bias against disconfirmatory evidence (BADE) is associated with schizotypy. Schizophrenia Research. 2007;90(1-3):334-7.

21. Elliott B, Joyce E, Shorvon S. Delusions, illusions and hallucinations in epilepsy: 2. Complex phenomena and psychosis. Epilepsy Research. 2009;85(2-3):172-86.

22. Fujii D, Ahmed I. Characteristics of Psychotic Disorder Due to Traumatic Brain Injury. Journal of Neuropsychiatry and Clinical Neuroscience. 2002;14(2):130-40.

23. Koponen S, Taiminen T, Portin R, Himanen L, Isoniemi H, Heinonen H, et al. Axis I and II Psychiatric Disorders After Traumatic Brain Injury: A 30-Year Follow-Up Study. American Journal of Psychiatry. 2002;159(8):1315-21.

24. Van Assche L, Van Aubel E, Van de Ven L, Bouckaert F, Luyten P, Vandenbulcke M. The Neuropsychological Profile and Phenomenology of Late Onset Psychosis: A Crosssectional Study on the Differential Diagnosis of Very-Late-Onset Schizophrenia-Like Psychosis, Dementia with Lewy Bodies and Alzheimer's Type Dementia with Psychosis. Archives of Clinical Neuropsychology. 5 ed. 2018;10(2):229.

25. Bersani G, Prevete E. Novel psychoactive substances (NPS) use in severe mental illness (SMI) patients: Potential changes in the phenomenology of psychiatric diseases. Human Psychopharmacology Clinical and Experimental. 2017;32(3):e2591.

McKetin R, Baker AL, Dawe S, Voce A, Lubman DI. Differences in the symptom profile of methamphetamine-related psychosis and primary psychotic disorders. Psychiatry Research. 2017;251:349-54.

Quinn CA, Wilson H, Cockshaw W, Barkus E, Hides L. Development and validation of the cannabis experiences questionnaire - Intoxication effects checklist (CEQ-I) short form. Schizophrenia Research. 2017;189:91-6.

28.

Nesse RM. Evolutionary Psychology and Mental Health. Hoboken, NJ, USA: John Wiley \& Sons, Inc; 2015. 


\section{3.}

\section{7.}

29. Boyer P, Firat R, van Leeuwen F. Safety, Threat, and Stress in Intergroup Relations. Perspectives on Psychological Science. 2015;10(4):434-50.

Oliver JE, Wood TJ. Conspiracy Theories and the Paranoid Style(s) of Mass Opinion. American Journal of Political Science. 2014;58(4):952-66.

31. Douglas KM, Sutton RM, Cichocka A. The Psychology of Conspiracy Theories. Current Directions in Psychological Science. 2017;26(6):538-42.

32. Harper DJ. Histories of suspicion in a time of conspiracy: a reflection on Aubrey Lewis's history of paranoia. History of the Human Sciences. 3rd ed. 1994 Jul 25;7(3):89-109.

Andreasen NC. SAPS - Scale for the Assessment of Positive Symptoms. University of Iowa; 1984.

34. Oyebode F. Sims' Symptoms in the Mind. Saunders Elsevier; 2008.

35. Kim K-I, Li D, Jiang Z, Xing Ji Cui, Lin L, Jiang Ju Kang, et al. Schizophrenic Delusions Among Koreans, Korean-Chinese and Chinese: a Transcultural Study. International Journal of Social Psychiatry. 1993;39(3):190-9.

Stompe T, Friedman A, Ortwein G, Strobl R, Chaudhry HR, Najam N, et al. Comparison of Delusions among Schizophrenics in Austria and in Pakistan. Psychopathology. 1999;32(5):225-34.

Green C, Garety PA, Freeman D, Fowler D, Bebbington P, Dunn G, et al. Content and affect in persecutory delusions. British Journal of Clinical Psychology. 2010;45(4):56177.

Boissy A. Fear and Fearfulness in Animals. Quarterly Review of Biology. 1995;70(2):165-91.

Smith BR, Blumstein DT. Fitness consequences of personality: a meta-analysis. Behavioral Ecology. 2008;19(2):448-55.

Sih A, Del Giudice M. Linking behavioural syndromes and cognition: a behavioural ecology perspective. Philosophical Transactions of the Royal Society of London B: Biological Sciences. 2012;367(1603):2762-72.

Haselton MG, Nettle D. The paranoid optimist: an integrative evolutionary model of cognitive biases. Personality and Social Psychology Review. 2006; 10: 47-66. of Psychopathology. Cham: Springer International Publishing; 2017. pp. 93-116. (Evolutionary Psychology; vol. 51).

Miloyan B, Bulley A, Suddendorf T. Anxiety: Here and Beyond. Emotion Review. 2018;10(4):175407391773857. ultimate perspectives. Henry JD, Addis DR, Suddendorf T, Rendell PG, editors. British Journal of Clinical Psychology. 2016;55(1):4-22.

45. Harcourt AH, de Waal F. Coalitions and Alliances in Humans and Other Animals. Oxford University Press; 1992. 
Bissonnette A, Perry S, Barrett L. Coalitions in theory and reality: a review of pertinent variables and processes. 2015; Behaviour; 152: 1-56.

47. Wrangham RW, Glowacki L. Intergroup Aggression in Chimpanzees and War in Nomadic Hunter-Gatherers. Human Nature. 2012;23(1):5-29.

48. Gershman B. Witchcraft beliefs and the erosion of social capital: Evidence from SubSaharan Africa and beyond. Journal of Development Economics. 2016;120:182-208.

49. Mace R, Thomas MG, Wu J, He Q, Ji T, Tao Y. Population structured by witcheraft beliefs. Nature Human Behaviour. 2018 ;2(1):39-44.

Vaillancourt T. Do human females use indirect aggression as an intrasexual competition strategy? Philosophical Transactions of the Royal Society of London B: Biological Sciences. 2013;368(1631):20130080-0.

51. Hess NH, Hagen EH. Sex differences in indirect aggression: Psychological evidence from young adults. Evolution and Human Behavior. 2006;27:231-45.

Sell A, Tooby J, Cosmides L. Formidability and the logic of human anger. Proceedings of the National Academy of Sciences USA; 2009;106(35):15073-8.

Tooby J, Cosmides L. Groups in Mind: The Coalitional Roots of War and Morality. Vol. 50, Human Morality and Sociality. London: Macmillan Education UK; 2010. 44 p.

Pietraszewski D, Cosmides L, Tooby J. The Content of Our Cooperation, Not the Color of Our Skin: An Alliance Detection System Regulates Categorization by Coalition and Race, but Not Sex. Plos ONE. 2014;9(2):e88534.

Pietraszewski D, Curry OS, Petersen MB, Cosmides L, Tooby J. Constituents of political cognition: Race, party politics, and the alliance detection system. Cognition. 2015;140:24-39.

Saalfeld V, Ramadan Z, Bell V, Raihani NJ. Experimentally induced social threat increases paranoid thinking. Royal Society Open Science.5(8):180569.

Greenburgh A, Bell V, J Raihani N. PsyArXiv Preprints | Paranoia and conspiracy: group cohesion increases harmful intent attribution in the Trust Game. https://psyarxiv.com/mgzjr/

Tajfel H, Turner J. An integrative theory of intergroup conflict. In: Austin W, Worchel $\mathrm{S}$, editors. The social Psychology of intergroup relations. Pacific Grove, CA: researchgate.net; 1979. pp. 33-48.

Liberman Z, Woodward AL, Kinzler KD. The Origins of Social Categorization. Trends In Cognitive Sciences. 2017;21(7):556-68.

Dunham Y. Mere Membership. Trends In Cognitive Sciences. 2018;22(9):780-93.

Otten S. The Minimal Group Paradigm and its maximal impact in research on social categorization. Current Opinion in Psychology. 2016;11:85-9.

Wood J, Dennard S. Gang Membership: Links to Violence Exposure, Paranoia, PTSD, Anxiety, and Forced Control of Behavior in Prison. Psychiatry. 3rd ed. Routledge; 2017 Apr 14;80(1):30-41. 
77.

63. Pizarro J, Silver RC, Prause J. Physical and Mental Health Costs of Traumatic War Experiences Among Civil War Veterans. Archives of General Psychiatry. 2006 ;63(2):193-200.

64. Kaštelan A, Frančišković T, Moro L, Rončević-Gržeta I, Grković J, Jurcan V, et al. Psychotic Symptoms in Combat-Related Post-Traumatic Stress Disorder. Military Medicine. 2007;172(3):273-7.

65. Ember CR, Adem TA, Skoggard I. Risk, Uncertainty, and Violence in Eastern Africa. Human Nature. 2012;24(1):33-58.

Anderson F, Freeman D. Socioeconomic Status and Paranoia. The Journal of Nervous and Mental Disease. 2013;201(8):698-702.

67. Wrangham RW. Evolution of coalitionary killing. American Journal of Physical Anthropology. 1999;110(S29):1-30.

68. Johnson DDP, MacKay NJ. Fight the power: Lanchester's laws of combat in human evolution. Evolution and Human Behavior. 2015;36(2):152-63.

Gayer-Anderson C, Morgan C. Social networks, support and early psychosis: a systematic review. Epidemiology and Psychiatric Sciences. 2013;22(2):131-46.

Wickham S, Taylor P, Shevlin M, Bentall RP. The Impact of Social Deprivation on Paranoia, Hallucinations, Mania and Depression: The Role of Discrimination Social Support, Stress and Trust. Plos ONE. 2014;9(8):e105140.

Shaikh M, Ellett L, Dutt A, Day F, Laing J, Kroll J. Perceived ethnic discrimination and persecutory paranoia in individuals at ultra-high risk for psychosis. Psychiatry Research. 2016;241:309-14.

Bosqui TJ, Hoy K, Shannon C. A systematic review and meta-analysis of the ethnic density effect in psychotic disorders. Social Psychiatry and Psychiatric Epidemiology. 2014;49(4):519-29.

Kessler RC, Amminger GP, Aguilar-Gaxiola S, Alonso J, Lee S, st n TB. Age of onset of mental disorders: a review of recent literature. Current Opinion in Psychiatry. 2007 ;20(4):359-64.

Freeman D, McManus S, Brugha T, Meltzer H, Jenkins R, Bebbington P. Concomitants of paranoia in the general population. Psychological Medicine. 2010;41(05):923-36.

Geary D. Evolution and development of boys' social behavior. Developmental Review. 2003;23(4):444-70.

Glowacki L, Isakov A, Wrangham RW, McDermott R, Fowler JH, Christakis NA. Formation of raiding parties for intergroup violence is mediated by social network structure. Proceedings of the National Academy of Sciences USA; 2016;113(43):12114-9.

Del Giudice M, Angeleri R, Manera V. The juvenile transition: A developmental switch point in human life history. Developmental Review. 2009;29(1):1-31. 


\section{9.} victimization in childhood and adolescence: A meta-analytic investigation. School Psychology Quarterly. 2010;25(2):65-83.

Volk AA, Camilleri JA, Dane AV, Marini ZA. Is Adolescent Bullying an Evolutionary Adaptation? Aggressive Behavior. 2012;38(3):222-38.

80. Blakemore S-J. Avoiding Social Risk in Adolescence. Current Directions in Psychological Science. 2018;096372141773814.

81. Spielberg JM, Olino TM, Forbes EE, Dahl RE. Exciting fear in adolescence: Does pubertal development alter threat processing? Developmental Cognitive Neuroscience. 2014;8:86-95.

82. Silk JS, Siegle GJ, Lee KH, Nelson EE, Stroud LR, Dahl RE. Increased neural response to peer rejection associated with adolescent depression and pubertal development. Social Cognitive and Affective Neuroscience. 2014;9(11):1798-807.

83. Paus T, Keshavan M, Giedd JN. Why do many psychiatric disorders emerge during adolescence? Nature Reviews Neuroscience. 2008;9(12):947-57.

84. Fawcett TW, Frankenhuis WE. Adaptive explanations for sensitive windows in development. Frontiers in Zoology. 2015;12 Suppl 1(Suppl 1):S3.

85. Frankenhuis WE, de Weerth C. Does Early-Life Exposure to Stress Shape or Impair Cognition? Current Directions in Psychological Science. 2013;22(5):407-12.

86. Frankenhuis WE, Nettle D, McNamara JM. Echoes of Early Life: Recent Insights From Mathematical Modeling. Child Development. 2018;6(Suppl. 1):769-15.

\section{7.}

English S, Browning LE, Raihani NJ. Developmental plasticity and social specialization in cooperative societies. Animal Behaviour. 2015;106:37-42.

88. Panchanathan K, Frankenhuis WE. The evolution of sensitive periods in a model of incremental development. Proceedings of the Royal Society B. 2016;283(1823):20152439.

89. Blakemore S-J. Development of the social brain during adolescence. The Quarterly Journal of Experimental Psychology. 2008;61(1):40-9.

Fuhrmann D, Knoll LJ, Blakemore S-J. Adolescence as a Sensitive Period of Brain Development. Trends In Cognitive Sciences. 2015;19(10):558-66.

91. Frankenhuis WE, Panchanathan K. Individual Differences in Developmental Plasticity May Result From Stochastic Sampling. Perspectives on Psychological Science. 2011;6(4):336-47.

Wakefield JC. The concept of Mental Disorder. American Psychologist. 1992;42:37388.

94. Bateson M, Brilot B, of DNTCJ, 2011. Anxiety: an evolutionary approach. The

Wakefield JC. Evolutionary versus prototype analyses of the concept of disorder. Journal of Abnormal Psychology. 1999;108(3):374-99. 


\section{6.}

Nettle D, Bateson M. The Evolutionary Origins of Mood and Its Disorders. Current Biology. 2012;22(17):R712-21.

Campbell MM, Sibeko G, Mall S, Baldinger A, Nagdee M, Susser E, et al. The content of delusions in a sample of South African Xhosa people with schizophrenia. BMC Psychiatry. 2017;17(1):41.

Tinbergen N. On aims and methods of Ethology. Ethology. 1963;20(4):410-33.

Mayr E. Proximate and ultimate causations. Biology \& Philosophy. 1993 Jan;8(1):93-4.

Mayr E. Cause and Effect in Biology. Science. 1961;134(3489):1501-6.

100. Scott-Phillips TC, Dickins TE, West SA. Evolutionary theory and the ultimateproximate distinction in the human behavioral sciences. Perspectives on Psychological Science. 2011, 6: 38-47.

101. Penn DC, psychology DPARO, 2007. Causal cognition in human and nonhuman animals: A comparative, critical review. Annual Reviews in Psychology 2007;58(1):97118.

102. Penn DC, Povinelli DJ. On the lack of evidence that non-human animals possess anything remotely resembling a "theory of mind." Philosophical Transactions of the Royal Society of London B: Biological Sciences. 2007;362(1480):731-44.

103. Penn DC, Holyoak KJ, Povinelli DJ. Darwin's mistake: Explaining the discontinuity between human and nonhuman minds. Behav Brain Sci. Cambridge University Press; 2008 Apr 1;31(2):109-30.

104. Stuart-Fox M. The origins of causal cognition in early hominins. Biology \& Philosophy. Springer Netherlands; 2014 Sep 6;30(2):247-66.

105. McKay R, Efferson C. The subtleties of error management. Evolution and Human Behavior 2010 Sep 1;31(5):309-19.

106. McNamara JM, Trimmer PC, Erikksson A, Marshall J, Houston AI. Environmental variability can select for optimism or pessimism. Ecology Letters. 2011 14: 58-62.

107. Marshall J, Trimmer PC, Houston AI, McNamara JM. On evolutionary explanations of cognitive biases. Trends in Ecology and Evolution. 2013 28: 469-473.

108. Trimmer PC. Optimistic and realistic perspectives on cognitive biases. Current Opinion in Behavioral Sciences. 2016;12:37-43.

109. McCullough ME, Kurzban R, Tabak BA. Cognitive systems for revenge and forgiveness. Behavioral and Brain Sciences. 2013;36(01):1-15.

110. Clutton-Brock TH, Parker GA. Punishment in animal societies. Nature. 1995;373(6511):209-16.

111. Raihani NJ, Thornton A, Bshary R. Punishment and cooperation in nature. Trends in Ecology and Evolution. 2012;27(5):288-95.

112. Robertson TE, Delton AW, Klein SB, Cosmides L, Tooby J. Keeping the benefits of group cooperation: domain-specific responses to distinct causes of social exclusion. Evolution and Human Behavior. 2014;35:472-80. 
113. Feinberg M, Willer R, Schultz M. Gossip and Ostracism Promote Cooperation in Groups. Psychological Science. 2014;25:656-64.

114. Delton AW, Krasnow M, Cosmides L, Tooby J. Evolution of direct reciprocity under uncertainty can explain human generosity in one-shot encounters. Proceedings of the National Academy of Sciences USA. 2011;108(32):13335-40.

115. Zimmermann J, Efferson C. One-shot reciprocity under error management is unbiased and fragile. Evolution and Human Behavior. 2017;38(1):39-47.

Raihani NJ, Bell V. Paranoia and the social representation of others: a large-scale game theory approach. Scientific Reports. Nature Publishing Group; 2017;7(1):4544.

Raihani NJ, Bell V. Conflict and cooperation in paranoia: a large-scale behavioural experiment. Psychological Medicine. 2017;76:1-11. 